\title{
Kalman 滤波用于色谱重胫峰的辨析*
}

\author{
石乐明1）楼蔓藤2）李梦龙3）林润国4）李志良 ${ }^{3)}$ \\ (1) 中国科学院化工冶金研究所,北京 100080；2) 广州分析测试中心,广州 510070; \\ ") 湖南大学化学化工系,长沙 410082；"广西化工研究所环保室,南宁 530000)
}

关链调 Kalman、色谱分析法、重叠峰辨析、拟合

色谱系使用最广泛的分析测试手段之一, 与光谱分析一样, 经常遇到谱图重叠的问题, 影 响分析测定. 对于部分重叠色谱峰可采用较直观的垂线法、切线法分辨; 但对于严重重叠峰, 这些方法往往效果不佳. 为此有的学者 ${ }^{[1,2]}$ 提出用函数拟合的分峰方法. 本文研究了 Kalman 滤波与拟合函数相结合的辨析新方法, 模拟及实测重叠峰分辨结果均表明本法具有较强解析 功能.

\section{一、原理与算法}

1. 拟合函数 文献中有以下几种常用函数:

(a) 高斯函数

$$
Z_{i}(t)=H \exp \left[-\frac{1}{2}\left(t-t_{i}\right)^{2} / \sigma^{2}\right]
$$

(b) 双高斯函数

$$
Z_{i}(t)=H \exp \left[-\frac{1}{2}\left(t-t_{i}\right)^{2} / \sigma_{i}^{2}\right],
$$

当 $t<t_{i}$ 时, $\sigma_{i}=\sigma_{l}$; 当 $t \geqslant t_{i}$ 时, $\sigma_{i}=\sigma_{r}$.

(c) 高斯函数的指数衰减修正模型 (EMG)

$$
Z_{i}(t)=H \exp \left(\frac{\sigma^{2}}{2 \tau}-\frac{t-t_{i}}{\tau}\right) \int_{-\infty}^{z} \exp \left(-x^{2}\right) d x,
$$

其中 $Z=\left(\frac{t-t_{i}}{\sigma}-\frac{\sigma}{\tau}\right) / \sqrt{2}, H=A /(\sqrt{\pi} \tau)$.

(d) 改进双高斯指数函数

$$
Z_{i}(t)= \begin{cases}H \exp \left\{-\frac{1}{2}\left[\left(t-t_{i}\right) / \sigma_{l}\right]^{\beta_{l}}\right\}=H / e^{\frac{1}{2}\left(\frac{t_{i}-t}{\sigma_{l}}\right)^{\beta_{l}}} & \left(t<t_{i}\right), \\ H \exp \left\{-\frac{1}{2}\left[\left(t_{i}-t\right) / \sigma_{r}\right]^{\beta_{r}}\right\}=H / e^{\frac{1}{2}\left(\frac{t-t_{i}}{\sigma_{r}}\right)^{\beta_{r}}}\left(t \geqslant t_{i}\right) .\end{cases}
$$

上述各式中, $Z_{i}(t)$ 为 $t$ 时刻的色谱信号; $H$ 为峰高; $t_{i}$ 为组分 $i$ 的色谱峰位保留值; $\sigma$ 为标 准偏差, 即色谱图曲线拐点与峰位间的距离; $\sigma_{t}$ 和 $\sigma r$ 分别峰前和峰后的标准偏差; $\tau$ 为指数

本文 1990 年 12 月 13 日收到. 1991 年 6 月 20 日收到修改稿.

- 中国科学院计算机化学实验室及国家自然科学基金资助呐目. 
衰减时间常数.

2. 多组分定青模型与 Kalman 滤波算法

对于满足线性加合性原理的色谱多组分定

量分析体系,其量测模型为:

$$
\begin{aligned}
Z(k) & =Y(k) X(k)+v(k)=y_{1 k}+y_{2 k}+\cdots+y_{n k}+v(k) \\
& =\sum_{i=1}^{n} y_{i k}+v(k)(i=1,2, \cdots, n ; k=1,2, \cdots, m),
\end{aligned}
$$

式中 $x_{i}$ 为组分 $i$ 的相对含量, $Z(k)$ 为保留值 $t_{k}$ 时的色谱信号值; $v(k)$ 为量测噪声, 通 常设为零均值白噪声, 其各处方差相等; $Y(k)$ 为组分 $i$ 在保留值 $t_{k}$ 时的色谱应答值组成的 量测系数矢量. 经 Kalman 滤波递推计算可以求出各组分含量的最佳估计值. 具体算法参 见文献 [3-5],并经作者适当简化 ${ }^{[5]}$.

3. 函数拟合分国适叠当函数形式拟合实际色谱峰，其吻合程度常借评价函数 来衡量. 依最小二乘原理, 选择拟合函数计算值与相应实测值的残差平方和为评价函数, 记作 $S(Q)$ :

$$
S(Q)=\sum_{k=1}^{m}\left[f_{k}(Q)\right]^{2} \rightarrow \min , \frac{\partial S(Q)}{\partial Q_{i}}=0 ;(j=1,2, \cdots, l \times n),
$$

此处 $l$ 为雷叠峰解析为单组分峰时拟合函数中待定参数的个数, $n$ 为组分数目.

\section{二、结果与 讨 论}

1. 模拟重叠峰的 Kalman 滤波分辩结果按式 (1)、(5) 并叠加一高斯噪声构造模 拟重叠峰数据, 用 Kalman 滤波方法确定各组分含量, 相对误差小于 $2 \%$, 且证明滤波初值及 测䜻噪声取值对滤波结果影响甚小.

2. 色谱峰的拟合与拟合函数的选择欲获得良好的色谱峰拟合效果, 关键是选择合适 的拟合函数. 其形式应满足以下要求:（a）对各种色谱峰形均具有较好的拟合功能; (b) 在 满足精确度要求前提下所包含的参数越少越好; (c) 各参数具有明确的物理意义, 以便确定 初值. 用前述四种拟合函数对对二甲苯气相色谱图进行了拟合, 结果表明, 采用高斯函数 (1) 式得不到正确结果; 采用双高斯函数 (2) 式时结果得到显著改善; 而采用 EMG (3) 式和改 进双高斯指数函数 (4) 式亦均能获得良好结果. 究其原因, 我们认为是 (2) 式通过调节 $\sigma_{1}$ 、 $\sigma_{r}$ 来拟合拖尾程度,但据 $\sigma_{t} 、 \sigma_{r}$ 的物理意义,它们不能偏离实际峰拐点至峰位间距离太大, 否 则会使峰形变宽或变窄, 使吻合程度反而降低; (3) 式亦较符合色谱实际情况 ${ }^{[2]} ;(4)$ 式则是 通过再调节 $\beta_{l} 、 \beta_{r}$ 来改变拟合曲线变化速度,常比 $\sigma_{l}, \sigma_{r}$ 灵敏得多, 从而获得良好的拟合结 果. 本文选用 (4) 式作为拟合函效.

拟合函数初值按常规是从重叠峰一阶或二阶导数(曲线)中选取, 此法受重叠程度制约很 大. 实验发现在满足线性加合性且实验条件相同时, 各组分在单独存在时与处于混合物中峰 形相似, 故取各组分纯色谱峰的参数值(除峰高 $H$ 外) 作为拟合解析的参数初值 (先单峰拟合, 优化参数). 四种体系的重叠峰拟合解析结果如图 1 所示。实验表明: (a) 本法对各体系分 辡拟合误差均小于 $7 \%$ ，优于高斯、双高斯、对数正态、 $\Gamma$ 函数等拟合法; (b) 受峰形限制小， 适用性强; (c) 迭代次数与计算量大为减少.

实测重叠峰的 Kalman 滤波解析结果: 采用 (4) 式及采用单位浓度各纯组分色谱标 准谱图作为量测系数 $y_{i k}$ (分别简称为拟合函数法及标准谱图法), 并经 Kalman 滤波方法对 


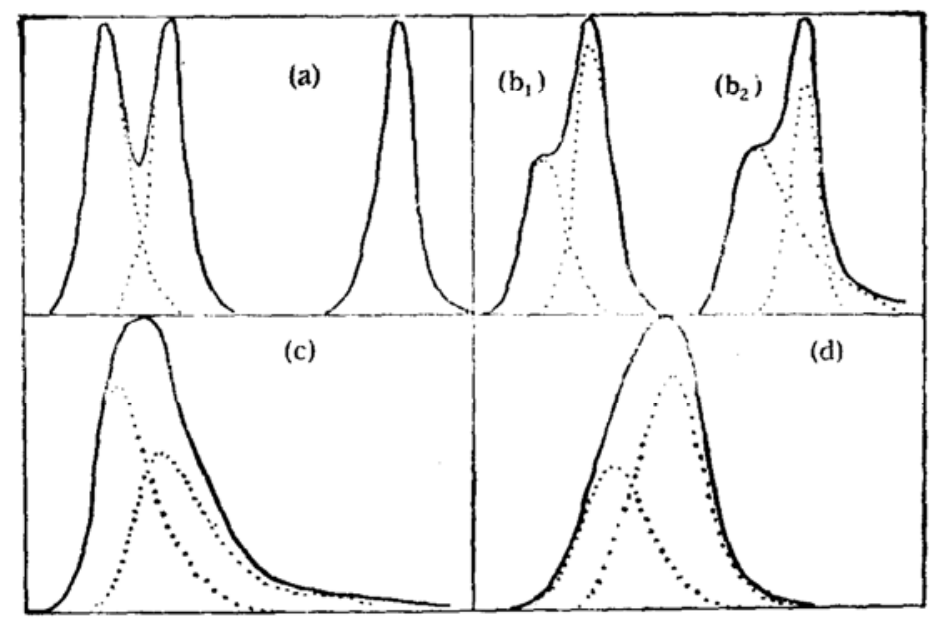

图 1 重叠色谱峰的改进双高斯指数函数拟合解析

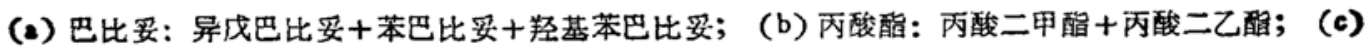
六六六: $\gamma-\mathrm{C}_{6} \mathrm{H}_{6} \mathrm{Cl}_{6}+\beta-\mathrm{C}_{6} \mathrm{H}_{6} \mathrm{Cl}_{6} ;$ (d) DDT: $o, p-\mathrm{DDT}+p, p^{\prime}-\mathrm{DDT}$.

实际色谱峰(一); 分辫单一峰 (......)

表 1 Kalman 滤波与改进双高斯指数函数拟合用于分辨重叠色谱峰

\begin{tabular}{|c|c|c|c|c|c|c|c|c|c|c|}
\hline \multirow{2}{*}{ 编号 } & \multirow{2}{*}{ 画叠峰体系 } & \multicolumn{3}{|c|}{ 实际组成* } & \multicolumn{3}{|c|}{ 标准图橧估计 } & \multicolumn{3}{|c|}{ 报合函数估计 } \\
\hline & & $x_{1}$ & $x_{2}$ & $\mathrm{X}_{3}$ & $\mathrm{x}_{1}$ & $\mathrm{X}_{2}$ & $x_{3}$ & $\mathrm{x}_{1}$ & $\mathrm{x}_{2}$ & $\mathrm{x}_{3}$ \\
\hline 1 & $\mathrm{X}_{1}=$ 异戊巴比罗 & 0.800 & 0.200 & 0.500 & 0.790 & 0.200 & 0.500 & 0.830 & 0.210 & 0.520 \\
\hline 2 & X $=$ 苯巴比罗 & 0.050 & 2.000 & 1.000 & 0.050 & 1.990 & 1.000 & 0.050 & 2.030 & 1.050 \\
\hline 3 & $\mathrm{X}_{3}=$ 羟基世比罗 & 2.000 & 0.400 & 1.000 & 1.980 & 0.410 & 1.020 & 2.010 & 0.420 & 1.030 \\
\hline 4 & & 0.500 & 0.500 & & 0.490 & 0.510 & & 0.480 & 0.490 & \\
\hline 5 & $X_{1}=$ 丙酸二甲酯 & 1.500 & 0.200 & & 1.500 & 0.200 & & 1.450 & 0.190 & \\
\hline 6 & $\mathrm{X}_{2}=$ 丙酸二乙醖 & 0.100 & 2.000 & & 0.100 & 1.990 & & 0.090 & 1.930 & \\
\hline 7 & & 0.200 & 0.900 & & 0.200 & 0.900 & & 0.210 & 0.930 & \\
\hline 8 & $\mathbf{X}_{1}=r-\mathrm{C}_{6} \mathrm{H}_{6} \mathrm{Cl}_{6}$ & 1.000 & 0.050 & & 1.030 & 0.050 & & 1.050 & 0.050 & \\
\hline 9 & $\mathrm{X}_{1}=\boldsymbol{\beta}-\mathrm{C}_{6} \mathrm{H}_{6} \mathrm{Cl}_{6}$ & 0.100 & 1.500 & & 0.100 & 1.480 & & 0.110 & 1.510 & \\
\hline 10 & & 2.000 & 0.100 & & 1.950 & 0.110 & & 2.020 & 0.110 & \\
\hline 11 & $\mathbf{X}_{1}=0, p-\mathrm{DDT}$ & 0.200 & 1.000 & & 0.210 & 0.970 & & 0.210 & 1.060 & \\
\hline 12 & $\mathrm{X}_{2}=p \cdot p^{\prime}-\mathrm{DDD}$ & 0.500 & 1.500 & & 0.510 & 1.480 & & 0.520 & 1.540 & \\
\hline
\end{tabular}

*均以相对含量表示.

实际色谱图重叠峰进行分辨解析, 先求各组分对各保留值处色谱讯号即峰高值 $Z(k)$ 的贡 献, 然后进行累加计算相应解析出的单一谱图面积再确定其组分含量(结果见表 1). 可知两 者结果一致,均能得到良好结果.

\section{参考文献}

[1] Grimalt, J., Iturriaga, H., Tomas, X., Anal. Chim. Acta, 139 (1982), 155.

[2] 赖聪、卢倨章、李浩春,色橧, 5(1987), 5: 271 .

[3] Brown, S.D., Anal. Chim. Acta, 181 (1986), 1.

[4] Hayashi, Y., Shibazaki, T., Matsuda, R. et al., J. Chromatogr., 407 (1987), 59.

[5] 本志良、石乐明、李梦龙,化学学报, 48(1990), 11: 1101 。

[6] 李志良、石乐明、李梦龙、林润国,色慻, 9(1991)，5: 308. 